\title{
Outcomes of metastatic gastric cancer in young adult patients treated with first-line combination chemotherapy
}

\author{
Hyun-Jun Kim¹, Kyoung Ha Kim², Seung Tae Kim³, Se Hoon Park ${ }^{3}$ \\ ${ }^{I}$ Department of Obstetrics and Gynecology, Konkuk University College of Medicine, Chungju, Korea \\ ${ }^{2}$ Department of Internal Medicine, Soonchunghyang University College of Medicine, Seoul, Korea \\ ${ }^{3}$ Department of Medicine, Sungkyunkwan University School of Medicine, Samsung Medical Center, Seoul, Korea
}

Received April 04, 2015; Revised August 15, 2015; Accepted August 25, 2015; Published Online September 20, 2015

\section{Original Article}

\begin{abstract}
Purpose: Despite conflicting data regarding survival after curative surgery, little is known about the prognosis of metastatic gastric cancer (MGC) in young adults. The current study was performed to determine whether younger age is an independent prognostic factor among MGC patients receiving first-line chemotherapy and to evaluate how age relates to other known prognostic parameters. Methods: The records of 1843 MGC patients who were consecutively treated with first-line combination chemotherapy at Samsung Medical Center (Seoul, Korea) between 2000 and 2007, including 570 patients aged 45 years or younger, were retrieved from a prospective cancer chemotherapy database. Results: In the younger group, there were significantly more bone metastases, ascites, poor performance status, low albumin, elevated alkaline phosphatase, and resections that were non-curative than in the older patients. Progression-free survival (PFS) and overall survival (OS) was shorter in younger patients (PFS, 4.2 months; OS, 7.1 months) than in older ones (PFS, 5.1 months; OS, 8.4 months). Nonetheless, younger age did not show an independent association with PFS or OS. Stratified analyses showed that younger age was related with poor outcome in the subgroups of good performance status and no bone metastasis. Conclusion: When matched for other prognostic factors, the prognosis of younger MGC patients receiving first-line combination chemotherapy does not differ from that of older patients. The poor survival of younger patients may be attributed to the association with other adverse prognostic factors.
\end{abstract}

Keywords: Gastric Cancer; Chemotherapy; Young Age

\section{Introduction}

Gastric cancer remains the most frequently occurring malignancy in Korea. ${ }^{1}$ Although more than half of patients are aged 70 years or more, ${ }^{2}$ some patients are diagnosed with gastric cancer at young age. ${ }^{3}$ Some studies have shown that gastric cancer in young adults occurs predominantly in women, with a high incidence of diffuse histology type, in contrast to older patients. ${ }^{3-5}$ Gastric cancer in young adults tends to be more advanced, ${ }^{6}$ mainly due to delayed diagnosis and more aggressive tumor behavior. However, the long-term survival after curative surgery depends on the stage of the disease, not on the age of the patient. ${ }^{7,8}$

For patients with advanced, recurrent, or metastatic gastric cancer (MGC), palliative chemotherapy is considered a standard of care in terms of survival and palliation of symptoms. ${ }^{9,10}$ While there have been advances in MGC treatment during the past decades, such as multi-drug combination chemotherapy, the obtained median survival times were limited to within 10 months. ${ }^{11,12}$ There is little data to support or refute the assertion that MGC is particularly aggressive in young adult patients. Although it is recognized that younger patients will better tolerate chemotherapy, we do not know whether younger MGC patients have a better prognosis or have a more aggressive disease than older ones. In an effort to define the prognosis of MGC in young adults, we conducted this retrospective study based on the data obtained from a prospective cancer registry. The current study was performed to determine whether younger age is an independent prognostic factor among MGC patients receiving first-line chemotherapy and to evaluate how age relates to other known prognostic parameters.

\section{Methods and Materials}

Our cancer chemotherapy database included 1897 consecutive patients with histologically confirmed MGC. Patients were eligible if they had been treated with taxanesand/or fluoropyrimidine-based first-line chemotherapy between 2000 and 2007. Fifty-four patients (3\%) were excluded from the analysis because they received single-agent chemotherapy, leaving 1843 patients in the 
study. The numbers of patients registered for this study each year and included in this analysis were 206, 203, 190, 230, 243, 233, 240, and 298 for the years 2000 through 2007, respectively. Patients who were enrolled in clinical trials were excluded in order to ensure the age was not a limitation for their chemotherapy. No prior chemotherapy or only adjuvant chemotherapy which had been completed more than 6 months prior to registration were allowed. All but the survival data was prospectively recorded. All patients provided written informed consent prior to receiving chemotherapy according to institutional guidelines, and the Samsung Medical Center (Seoul, Korea) institutional review board (IRB) reviewed and approved this study.

The most commonly used first-line chemotherapy was fluoropyrimidine \pm leucovorin and cisplatin $(\mathrm{n}=888)$, followed by the taxane and cisplatin combination $(\mathrm{n}=716)$, FOLFOX (leucovorin/fluoropyrimidine and oxaliplatin, $\mathrm{n}=$ 83), FOLFIRI (leucovorin/fluoropyrimidine and irinotecan, $n$ = 52), ECF (epirubicin, cisplatin and fluoropyrimidine, $\mathrm{n}=$ $45)$, and others $(n=59)$. Chemotherapy was repeated every 2 or 3 weeks according to the regimen. Clinical responses to chemotherapy were evaluated every 2 or 3 courses of chemotherapy, according to the response evaluation criteria for solid tumors (RECIST). ${ }^{13}$ The date of starting chemotherapy was used to calculating progression-free survival (PFS) and overall survival (OS). PFS was defined the time between the starting chemotherapy and the date on which disease progressed or the date on which the patient died. Time to death, whatever the cause, was used to calculate OS.

Patients were divided into two groups: younger patients $(\leq 45$ years old) and older ( $>45$ years old). The age limits for the two groups was determined using receiver operating characteristic (ROC) curves. The Kaplan-Meier method was used to estimate PFS and OS, and a log-rank test was used to test the statistical significance of differences between the two groups. In addition, multivariate Cox regression models were employed to examine the impact of clinical and treatment parameters on the outcomes of chemotherapy. Covariates selected were mostly based on our previous prognostic model study ${ }^{14}$, which included age (45 years or less v older), gender, previous gastrectomy, disease status (primary metastatic $\mathrm{v}$ recurrent), an Eastern Cooperative Oncology Group (ECOG) performance status ( $0-1 \mathrm{v} 2$ or more), number of involved sites (one $\mathrm{v} 2$ or more), sites of metastases, presence of ascites, baseline chemistry profiles, and hemoglobin level. Laboratory parameters were initially recorded as continuous variables and later dichotomized according to the median value of each variable. The potential presence of interaction effects between age and other clinical parameters was tested by defining product terms for the respective factors in a regression model. $P$-values of $<0.05$ were considered significant.

TABLE 1: Patient characteristics according to their age.

\begin{tabular}{|c|c|c|c|c|}
\hline & All patients $(\mathrm{n}=1843)$ & Younger $(n=570)$ & Older $(n=1273)$ & $P$ \\
\hline Median age (range), years & $55(22-83)$ & $40(22-45)$ & $60(46-83)$ & \\
\hline Male gender & 1200 & 273 & 927 & $<0.01$ \\
\hline ECOG performance status & & & & $<0.01$ \\
\hline $0-1$ & 1548 & 455 & 1093 & \\
\hline 2 or more & 295 & 115 & 180 & \\
\hline Disease status & & & & $<0.01$ \\
\hline Primary metastatic & 1256 & 414 & 842 & \\
\hline Recurrent after surgery & 587 & 156 & 431 & \\
\hline Lauren classification & & & & $<0.01$ \\
\hline Diffuse & 471 & 201 & 270 & \\
\hline Intestinal & 1360 & 365 & 995 & \\
\hline Mixed or unknown & 12 & 4 & 8 & \\
\hline Prior gastrectomy & 975 & 269 & 706 & $<0.01$ \\
\hline Number of involved site(s) & & & & 0.07 \\
\hline One & 387 & 105 & 282 & \\
\hline Two or more & 1456 & 465 & 991 & \\
\hline \multicolumn{5}{|l|}{ Metastatic site } \\
\hline Liver & 518 & 189 & 429 & 0.22 \\
\hline Lung & 167 & 37 & 130 & 0.01 \\
\hline Bone & 127 & 54 & 73 & $<0.01$ \\
\hline Ascites & 506 & 206 & 300 & $<0.01$ \\
\hline $\begin{array}{l}\text { Hemoglobin, g/L } \\
\text { Median (range) }\end{array}$ & $11.3(3.2-17.8)$ & $11.4(3.2-17.8)$ & $11.3(5.5-16.7)$ & 0.62 \\
\hline \multicolumn{5}{|l|}{ Chemistry (median, range) } \\
\hline Albumin, g/dL & $3.7(0.3-5.0)$ & $3.7(1.8-4.9)$ & $3.6(0.3-5.0)$ & 0.16 \\
\hline Bilirubin, mg/dL & $0.5(0.1-17.7)$ & $0.5(0.1-8.2)$ & $0.5(0.1-17.7)$ & 0.67 \\
\hline Alkaline phosphatase, U/L & $85(9-3532)$ & $89(9-2180)$ & $75(29-3532)$ & $<0.01$ \\
\hline Calcium, mg/dL & $8.9(6.8-14.4)$ & $8.9(6.9-13.4)$ & $8.9(6.8-14.4)$ & 0.21 \\
\hline
\end{tabular}


TABLE 2: Univariate analysis of survival according to baseline clinical parameters.

\begin{tabular}{|c|c|c|c|c|}
\hline & $\mathrm{n}$ & OS, mo & HR $(95 \%$ CI $)$ & $P$ \\
\hline Age & & & & $<0.01$ \\
\hline$\leq 45$ years & 570 & 7.1 & 1.00 & \\
\hline$>45$ years & 1273 & 8.4 & $0.83(0.75-0.91)$ & \\
\hline Gender & & & & 0.32 \\
\hline Male & 1200 & 8.0 & 1.00 & \\
\hline Female & 643 & 7.9 & $1.05(0.95-1.16)$ & \\
\hline Prior gastrectomy & & & & $<0.01$ \\
\hline No & 868 & 6.9 & 1.00 & \\
\hline Yes & 975 & 9.1 & $0.68(0.62-0.75)$ & \\
\hline Disease status & & & & $<0.01$ \\
\hline Primary metastatic & 1012 & 7.3 & 1.00 & \\
\hline Recurrent & 831 & 9.1 & $0.77(0.70-0.85)$ & \\
\hline Lauren classification & & & & 0.39 \\
\hline Diffuse or mixed & 483 & 7.9 & 1.00 & \\
\hline Intestinal & 1360 & 8.0 & $0.96(0.86-1.06)$ & \\
\hline No. of involved site(s) & & & & $<0.01$ \\
\hline One & 399 & 10.9 & 1.00 & \\
\hline Two or more & 1444 & 7.2 & $1.55(1.37-1.74)$ & \\
\hline Performance status & & & & $<0.01$ \\
\hline $0-1$ & 1548 & 9.0 & 1.00 & \\
\hline 2 or more & 295 & 3.7 & $2.40(2.11-2.72)$ & \\
\hline Albumin & & & & $<0.01$ \\
\hline$\leq 3.7 \mathrm{~g} / \mathrm{dL}$ & 1050 & 6.5 & 1.00 & \\
\hline$>3.7 \mathrm{~g} / \mathrm{dL}$ & 782 & 9.9 & $0.66(0.60-0.73)$ & \\
\hline Alkaline phosphatase & & & & $<0.01$ \\
\hline$\leq 85 \mathrm{U} / \mathrm{L}$ & 928 & 9.6 & 1.00 & \\
\hline$>85 \mathrm{U} / \mathrm{L}$ & 925 & 6.5 & $1.34(1.21-1.47)$ & \\
\hline Bilirubin & & & & 0.01 \\
\hline$\leq 0.5 \mathrm{mg} / \mathrm{dL}$ & 1021 & 8.6 & 1.00 & \\
\hline$>0.5 \mathrm{mg} / \mathrm{dL}$ & 816 & 6.8 & $1.22(1.11-1.35)$ & \\
\hline Calcium & & & & 0.17 \\
\hline$\leq 8.9 \mathrm{mg} / \mathrm{dL}$ & 973 & 8.5 & 1.00 & \\
\hline$>8.9 \mathrm{mg} / \mathrm{dL}$ & 800 & 7.5 & $1.07(0.97-1.18)$ & \\
\hline Hemoglobin & & & & 0.52 \\
\hline$\leq 11.3 \mathrm{~g} / \mathrm{L}$ & 927 & 7.8 & 1.00 & \\
\hline$>11.3 \mathrm{~g} / \mathrm{L}$ & 914 & 8.1 & $0.97(0.89-1.06)$ & \\
\hline Liver metastasis & & & & 0.01 \\
\hline No & 1328 & 8.4 & 1.00 & \\
\hline Yes & 515 & 7.5 & $1.15(1.03-1.27)$ & \\
\hline Lung metastasis & & & & 0.01 \\
\hline No & 171 & 8.1 & 1.00 & \\
\hline Yes & 124 & 6.5 & $1.49(1.27-1.75)$ & \\
\hline Bone metastasis & & & & $<0.01$ \\
\hline No & 1716 & 8.3 & 1.00 & \\
\hline Yes & 127 & 4.9 & $1.72(1.45-2.08)$ & \\
\hline Ascites & & & & $<0.01$ \\
\hline No & 1377 & 9.9 & 1.00 & \\
\hline Yes & 506 & 4.1 & $2.70(2.42-3.00)$ & \\
\hline
\end{tabular}

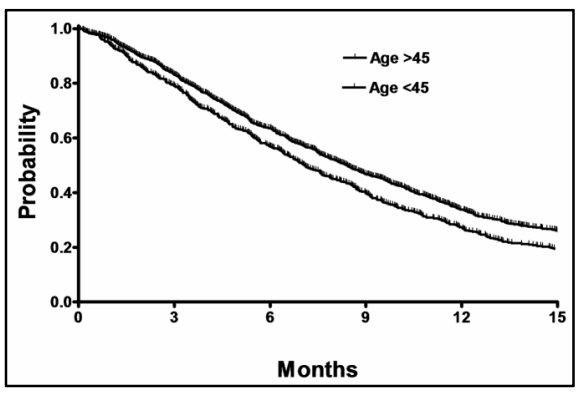

FIG. 1: Overall survival according to age. 


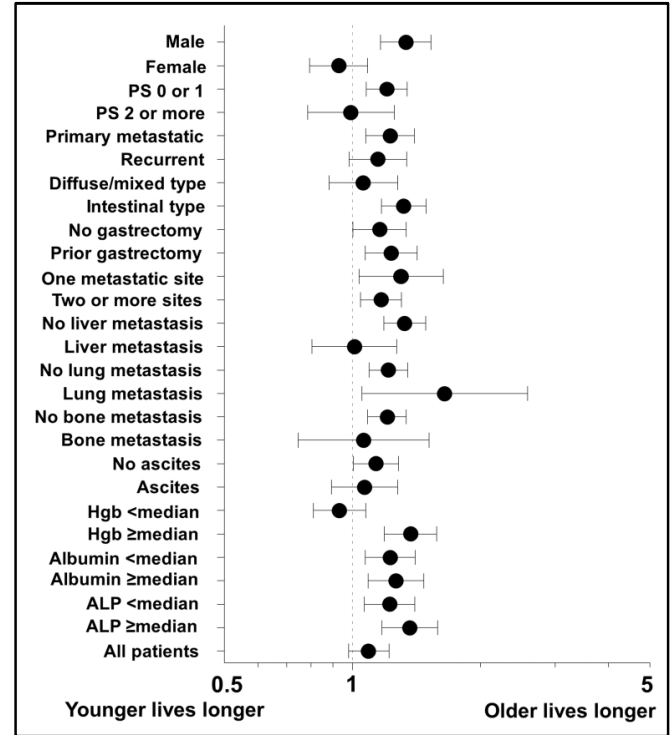

FIG. 2: Forest plot of overall survival.

TABLE 3: Multivariate analysis of survival according to baseline clinical parameters.

\begin{tabular}{lccc}
\hline \hline & HR & 95\% CI & $P$ \\
\cline { 2 - 4 } Age $\leq 45$ years & 1.09 & $0.98-1.22$ & 0.12 \\
No prior gastrectomy & 1.28 & $1.14-1.43$ & $<0.01$ \\
Primary metastatic disease & 1.04 & $0.94-1.16$ & 0.46 \\
Multiple involved sites & 1.01 & $0.88-1.16$ & 0.87 \\
Poor performance status $(2$ or & 1.83 & $1.60-2.09$ & $<0.01$ \\
more) & & & \\
Albumin $\leq 3.7$ g/dL & 1.28 & $1.16-1.41$ & $<0.01$ \\
Alkaline phosphatase $>85 \mathrm{U} / \mathrm{L}$ & 1.37 & $1.24-1.52$ & $<0.01$ \\
Bilirubin $>0.5 \mathrm{mg} / \mathrm{dL}$ & 1.08 & $0.98-1.19$ & 0.06 \\
Liver metastasis & 1.03 & $0.90-1.18$ & 0.25 \\
Lung metastasis & 1.01 & $0.83-1.22$ & 0.93 \\
Bone metastasis & 1.64 & $1.35-1.99$ & $<0.01$ \\
Ascites & 2.48 & $2.20-2.80$ & $<0.01$ \\
\hline \hline
\end{tabular}

\section{Results}

Thirty-one percent of patients $(n=570)$ were aged 45 years or less. Patient characteristics are given in Table 1 . The younger group had a larger proportion of women $(P<0.01)$, poor performance status $(P<0.01)$, no prior gastrectomy $(P<$ $0.01)$, diffuse type histology $(P<0.01)$, metastases to lung $(P$ $=0.01)$ or bone $(P<0.01)$, presence of ascites $(P<0.01)$, and elevated alkaline phosphatase level $(P<0.01)$ than the older group. In order to further clarify the association of age with these parameters, we performed a logistic regression analysis. This analysis showed an independent association of age with gender (odds ratio [OR], 0.42; 95\% confidence interval [CI], 0.34-0.53; $P<0.01$ ), prior gastrectomy (OR, 0.68; 95\% CI, $0.55-0.84 ; P<0.01$ ), performance status (OR, 0.73; 95\% CI, 0.55-0.97; $P=0.03$ ), bone metastasis (OR, 0.52; 95\% CI, 0.35-0.78; $P<0.01$ ), alkaline phosphatase (OR, 1.38; 95\% CI, 1.11-1.73; $P=<0.01$ ), and ascites (OR, 0.64; 95\% CI,
0.51-0.81; $P<0.01$ ), while the association with lung metastasis was just outside the limit of statistical significance (OR, 1.61; 95\% CI, 1.00-2.61; $P=0.05$ ). Thus, younger MGC patients tended to be at higher risk for death and/or progression because of these poor prognostic features. No significant differences were found with regard to hemoglobin level, Lauren classification, serum albumin, bilirubin, or calcium.

The median follow-up duration for all patients was 93.5 months. Of the 1843 patients analyzed in the study, 1795 (98\%) died. The estimated median PFS and OS were 4.8 months (95\% confidence interval [CI], 4.2-5.3 months) and 7.9 months (95\% CI, 7.5-8.4 months), respectively. Six-month and 12-month PFS were $40.3 \%$ and $15.5 \%$, respectively. PFS was shorter, although statistically insignificant, for younger MGC patients (median, $4.2 \mathrm{v} 4.9$ months; $P=0.08$ ) than for older group. OS at 6-month and 12 -month were $61.4 \%$ and $61.7 \%$, respectively. OS was significantly shorter for younger patients $(7.1 \mathrm{v} 8.4$ months; $P<0.01)$. The Kaplan-Meier estimate of OS is illustrated in Figure 1. In the univariate analysis, poor performance status (ECOG scale 2 or more), multiple metastatic sites, prior gastrectomy, primary metastatic disease, low albumin, elevated alkaline phosphatase, elevated bilirubin, metastases to liver, lung, and bone, the presence of ascites, as well as younger age, were adversely affected OS with statistical significance (Table 2). However, using multivariate techniques to adjust for differences in clinical parameters between younger and older patients, we found no significant association between younger age and OS (Table 3). Based on the proportional-hazards regression model, which included parameters for prior gastrectomy, disease status, multiple metastatic sites, performance status, metastases to liver, lung, peritoneum and bone, albumin, alkaline phosphatase, and bilirubin level, the relative risk for death among younger patients was 1.09 (95\% CI, $0.98-1.22 ; P=0.12)$ compared with that of the older MGC patients (Figure 2). Use of age as a continuous variable, rather than the dichotomous one, provided similar result.

Due to the disparity in a number of baseline parameters, we performed a secondary subgroup analysis for gender, performance status, prior gastrectomy, metastases to liver, lung, or bone, presence of ascites, and alkaline phosphatase level. As expected, OS in patients with poor performance status was short in both groups ( $3.3 \mathrm{v} 3.5$ months; $P=0.83$ ). However, younger patients with good performance status had a significantly shorter OS (8.4 months) than older patients with good performance status ( 9.5 months; $P<0.01$ ). A similar analysis in the subgroup of no bone metastasis showed a significant difference in OS (7.4 v 8.6 months; $P<$ 0.01). Shorter OS was observed in younger patients than older ones regardless of their albumin or alkaline phosphatase levels. 


\section{Discussions}

The present analysis of 1843 MGC patients who were treated with first-line chemotherapy has demonstrated a strong association between some baseline parameters, including performance status, baseline albumin and alkaline phosphatase levels, no prior gastrectomy, bone metastasis, and the presence of ascites, and OS. The younger patients aged $\leq 45$ demonstrated shorter OS than their older counterparts, yet in the multivariate analysis, no significant difference in the risk of death between younger and older patient (HR, 1.09; 95\% CI, $0.98-1.22 ; P=0.12$ ). One possible explanation for our observation is the association with other prognostic parameters.

There are conflicting results on the prognosis of gastric cancer in young adults. Some studies have shown a poor prognosis as a result of rapid progression of the disease in younger patient, ${ }^{4,5}$ while others have found no direct relationship, indicating that outcome was related to stage at diagnosis irrespective of age. , $7,8,15$ Our results differ from previous reports in two important respects. Firstly, only patients with advanced, inoperable, or metastatic disease were included. All patients received combination chemotherapy for their MGC. Secondly, data from all consecutive patients except for those enrolled in clinical trials were analyzed, in order to better reflect the patients seen in routine clinical practice.

In the current study, the younger patients had a larger proportion of poor performance status, no prior gastrectomy, metastases to liver, lung, or bone, presence of ascites, low albumin, and elevated alkaline phosphatase level than the older group. These observations come in accordance with those of previous studies. ${ }^{14,16}$ The nature of this association is not clear. Poor performance status may be attributed to poor tolerance of chemotherapy or more toxicity. Such interaction has also been reported in a recent retrospective analysis on 1299 Korean patients with gastric cancer, ${ }^{17}$ in which younger female patients had more undifferentiated tumors resulting in an unfavorable prognosis. It is still plausible that still unidentified differences in tumor biology that could originate from genetic aspects of the disease may play a more important role in the outcome of younger MGC patients. When interpreting the results, it is of note that this analysis represents only a small sample of patients and one-thirds of them were aged 45 years or younger at presentation. A meta-analysis of two large phase III trials comparing fluoropyrim-dine/cisplatin chemotherapy regimens in MGC patients, ${ }^{18,19}$ poor performance status, metastatic disease and age $<60$ were independent predictors of poor PFS and OS. ${ }^{20}$

\section{Conclusion}

In conclusion, multivariate analysis of a group of 1843 MGC patients treated with first-line combination chemotherapy demonstrated that poor performance status, no prior gastrectomy, low albumin, elevated alkaline phosphatase, bone metastasis, and the presence of ascites are associated with a shorter OS. MGC patients aged 45 years or younger had a worse OS than older patients; however, younger age was not found to be an independent prognostic parameter of OS in this study. The observed association of young age with established prognostic parameters may be the reason for this result. Furthermore, emerging science and the knowledge of disease may further guide us to develop individualized treatment for MGC patients.

\section{Conflict of interest}

The authors declare that they have no conflicts of interest. The authors alone are responsible for the content and writing of the paper.

\section{References}

1. Bae JM, Won YJ, Jung KW, et al. Annual report of the Korean central cancer registry program 2000: Based on Registered Data from 131 Hospitals. Cancer Res Treat. 2002;34:77-83.

2. Wanebo HJ, Kennedy BJ, Chmiel J, et al. Cancer of the stomach. A patient care study by the American College of Surgeons. Ann Surg. 1993;218:583-92.

3. Eguchi T, Takahashi Y, Yamagata M, et al. Gastric cancer in young patients. J Am Coll Surg. 1999;188:22-6.

4. Lai IR, Lee WJ, Chen CN, et al. Gastric cancer in the young. Hepatogastroenterology. 1997;44:1641-5.

5. Matley PJ, Dent DM, Madden MV, et al. Gastric carcinoma in young adults. Ann Surg. 1988;208:593-6.

6. Kokkola A, Sipponen P. Gastric carcinoma in young adults. Hepatogastroenterology. 2001;48:1552-5.

7. Santoro R, Carboni F, Lepiane P, et al. Clinicopathological features and prognosis of gastric cancer in young European adults. Br J Surg. 2007;94:737-42.

8. Llanos O, Butte JM, Crovari F, et al. Survival of young patients after gastrectomy for gastric cancer. World J Surg. 2006;30:17-20.

9. Pyrhonen S, Kuitunen T, Nyandoto P, et al. Randomised comparison of fluorouracil, epidoxorubicin and methotrexate (FEMTX) plus supportive care with supportive care alone in 
patients with non-resectable gastric cancer. $\mathrm{Br} \mathrm{J}$

Cancer. 1995;71:587-91.

10. Glimelius B, Ekstrom K, Hoffman K, et al. Randomized comparison between chemotherapy plus best supportive care with best supportive care in advanced gastric cancer. Ann Oncol. 1997;8:163-8.

11. Wagner AD, Grothe W, Haerting J, et al. Chemotherapy in advanced gastric cancer: a systematic review and meta-analysis based on aggregate data. J Clin Oncol. 2006;24:2903-9.

12. Ohtsu A, Yoshida S, Saijo N. Disparities in gastric cancer chemotherapy between the East and West. J Clin Oncol. 2006;24:2188-96.

13. Therasse P, Arbuck SG, Eisenhauer EA, et al. New guidelines to evaluate the response to treatment in solid tumors. European Organization for Research and Treatment of Cancer, National Cancer Institute of the United States, National Cancer Institute of Canada. J Natl Cancer Inst. 2000;92:205-16.

14. Lee J, Lim T, Uhm J, et al. Prognostic model to predict survival following first-line chemotherapy in patients with metastatic gastric adenocarcinoma. Ann Oncol. 2007;18:886-91.

15. Nakamura K, Ueyama T, Yao T, et al. Pathology and prognosis of gastric carcinoma. Findings in 10,000 patients who underwent primary gastrectomy. Cancer. 1992;70:1030-7.
16. Chau I, Norman AR, Cunningham D, et al. Multivariate prognostic factor analysis in locally advanced and metastatic esophago-gastric cancer--pooled analysis from three multicenter, randomized, controlled trials using individual patient data. J Clin Oncol. 2004;22:2395-403.

17. Kim JH, Boo YJ, Park JM, et al. Incidence and long-term outcome of young patients with gastric carcinoma according to sex: does hormonal status affect prognosis? Arch Surg. 2008;143:1062-7; discussion 1067.

18. Cunningham D, Starling N, Rao S, et al. Capeci tabine and oxaliplatin for advanced esophagogas tric cancer. NEngl J Med. 2008;358:36-46.

19. Kang YK, Kang WK, Shin DB, et al. Capecitabi ne/cisplatin versus 5-fluorouracil/cisplatin as first -line therapy in patients with advanced gastric cancer: a randomised phase III non-inferiority trial. Ann Oncol. 2009;20:666-73.

20. Okines AF, Norman AR, McCloud P, et al. Me ta-analysis of the REAL-2 and ML17032 trials: evaluating capecitabine-based combination chem otherapy and infused 5-fluorouracil-based combi nation chemotherapy for the treatment of adva nced oesophago-gastric cancer. Ann Oncol. 200 9;20:1529-34. 